\title{
Maximizing the Influence of the Innovative Products Diffusion considering Advertisement and Promotion Strategies
}

\author{
Liang'an Huo $\mathbb{D}^{1}{ }^{1}$ Jianbo $\mathrm{Xu},{ }^{1}$ Jianjia $\mathrm{He},{ }^{1}$ and Tingting Lin $^{2}$ \\ ${ }^{1}$ Business School, University of Shanghai for Science and Technology, Shanghai 200093, China \\ ${ }^{2}$ School of Electrical and Information Engineering, Shanghai Jiao Tong University, Shanghai 200240, China \\ Correspondence should be addressed to Liang'an Huo; huohuolin@yeah.net
}

Received 14 April 2021; Accepted 17 August 2021; Published 31 August 2021

Academic Editor: A. E. Matouk

Copyright ( 2021 Liang'an Huo et al. This is an open access article distributed under the Creative Commons Attribution License, which permits unrestricted use, distribution, and reproduction in any medium, provided the original work is properly cited.

With the acceleration of product updates and the intensification of product competition, product market strategies become the primary consideration for the enterprises, and the advertisement and promotion strategies are considered the two important strategies implemented by enterprises. This paper considers the enterprises of similar products and substitute, their formation of a competition between traditional products and innovative products, and establishes a mixed node-level information diffusion model to describe the dynamic product diffusion process with complex network theories. We implement advertising strategies for potential buyers who have not obtained product information and implement promotional strategies for those who have obtained product information. In accordance with Pontryagin maximization principle, we seek the best strategy to maximize the impact of innovative products and use numerical calculations to simulate the diffusion state of products. We found that the advertisement strategies play a decisive role in the marketing of innovative products. If product promotion strategies are added, the spread of innovative products will be more effective and more influential.

\section{Introduction}

With the development of science and technology, the way of dissemination of product information has undergone tremendous changes. Product performance has been gradually replaced by innovative product information marketing, and product diffusion has evolved into product information diffusion. Developing new product and accelerating the update of product are important strategies for enterprise to continuous operation and development, and advertisement and promotion strategies have become an important means of product competition. How to use advertisement and promotion strategies more effectively to improve the influence of innovative products has become a critical problem faced by contemporary enterprises.

The Bass model is one of the most well-known and widely used models of the new product diffusion model [1]. The researchers then made a series of assumptions around the Bass model and formed many improved models of the Bass model, such as time-varying parameter models $[2,3]$, repeated purchase diffusion model, mathematical model of time delay, new technology to describe the diffusion process [4], and multiple influence model [5]. Bertotti and Modanese used the results of the developed Bass innovation diffusion model based on the mean field theory with the heterogeneous network to calculate the secondary peak of diffusion [6]. Batista da Silva used the Bass model to predict the quantitative method of distributed photovoltaic system adoption rate [7]. Dev et al. established the classic Bass model of innovation diffusion to study the impact of product diffusion dynamics on the economic and environmental performance of inventory and production planning systems [8]. Kumar et al. proposed a nonlinear modification of the Bass model [9]. Massiani and Gohs studied the Bass model coefficients to predict the diffusion of innovative products [10]. Glassman and Kornelis used a random Bass model to predict product sales [11]. Mitra called for timely forecasts at the takeoff stage by using appropriate estimation techniques for the Bass 
model in the context of subsistence markets [12]. In addition to the Bass model, scholars have established a variety of models; Kim et al. proposed an agent-based diffusion model to study product diffusion in the highly competitive automotive market [13]. Aquino studied information transmission characteristics similar to complex systems [14]. Peers et al. used the seasonal diffusion model of information to propose a closed-form solution [15]. Galeotti et al. studied the multiperson communication model [16]. Iyengar et al. studied how opinion leaders and social crises in social networks influence the adoption of new products [17]. Tashiro considered the fashion sensitivity difference of the product diffusion model [18]. Considering the influence of heterogeneous social networks, Lopez-Pinto built a product diffusion model in a complex social network and obtained a threshold for the propagation speed $[19,20]$. $\mathrm{Li}$ and Jin considered the proliferation of single products in heterogeneous consumer social networks under the influence of mass media during the decision-making stage [21].

The essence of advertisement is to spread product information; it helps people have a better understanding about products' detail. Horsky and Simon examined the effects of advertisement on the sales growth of new infrequently purchased products [22]. Swami and Dutta conducted a numerical study on the best advertisement strategy for Japanese electronic products in the Indian market and obtained the best advertisement strategy with a higher initial advertisement investment and subsequent reduced advertisement investment [23]. Huang and Zhang studied the diffusion dynamics of two competing products with repeated buying behaviors in heterogeneous consumer social networks [24]. Tuli et al. proposed how people treat two products in two different patches and proposed a six-unit innovation diffusion model for two different patches [25]. Fu et al. proposed the propagation dynamics of competitive information on Internet social networks and proposed an improved SIR model for two types of competitive information [26]. Kermani et al. combined user heterogeneity, message content, and network structure and proposed a novel competitive influence model [27]. Christian et al. proposed an agentbased model that can handle repeated purchase decisions, solve the competitive diffusion of multiple products, and consider the spatial and temporal dimensions of innovation diffusion [28]. Zhao et al. presented the optimality system to solve the optimal control problems [29].

It can be found from previous research that most scholars have considered various factors related to the dissemination of product information, but few people consider the whole dynamic process of product disseminate from obtaining advertisement information to product purchase. Advertisement and promotion are two important means for companies to expand the market for their products. Some people may change their willingness to buy when they receive advertisements and promotional information and finally agree with the company's products under the inducement of the information.
We owe the augments to existing literatures; it has inspired this report. Compared with the results reported in this paper, there are different aspects that can be summarized as follows.

1 Angles: The existing literature considers the same kind of research from a perspective. In this paper, we proposed the implementation of advertisement and promotion strategies for alternative products, and it studied the two types of products from two perspectives.

(2) Background: The research background of this article is product information dissemination. As far as the author knows, there is little research on this aspect in the existing literatures.

(3) Models: The model is more complex in this paper; there are consider six states. We studied the first implementation of advertising strategies for potential buyers who do not know the product information and then the implementation of promotional strategies for those who already know the product information and become product disseminators. Its objective is to maximize the impact of innovative products.

(4) Algorithms: We used OS-FBS algorithm to solve the problem of advertisement-promotion strategy.

(5) Conclusions: In this article, the static optimal control variables are employed to provide companies with some suggestions, which are easier to implement.

This paper considers applying the advertisement promotion model to describe the dynamic process of competition between traditional alternative products and innovative products and establishes a mixed information node-level diffusion model based on complex network theories. We implement innovative product advertisement strategies for potential buyers who do not have access to product information and implement innovative product promotion strategies for those who have obtained innovative products. Eventually, they will be transformed into buyers of innovative products. Finally, based on Pontryagin maximum principle, seek strategies to maximize the influence of innovative products.

This paper is organized as follows. In Section 2, advertisement and promotion strategies are considered in the mixed diffusion model of traditional products and innovative products. In Section 3, we clearly show the existence of a method for solving the advertisement and promotion strategies. Numerical examples are given in Section 4. Finally, Section 5 presents conclusions for this article.

\section{The Modeling of the Advertisement and Promotion Problem}

It is assumed that product information is disseminated among netizens through online social networks (OSNs). Company goals are to maximize the influence of innovative 
products. Companies hope that, under the premise of relatively low costs, there are more innovative product disseminators and innovative product buyers to achieve the purpose of maximizing profits.

2.1. Preliminary Terms and Notations. Consider a population of $N$ persons labeled 1 through $N$ who are interconnected by OSNs. Let $\mathbf{A}=\left(a_{i j}\right)_{N \times N}$ denote the adjacency matrix of the underlying communication network of the population, i.e., $a_{i j}=1$ or 0 according to whether person $j$ can send messages directly to person $i$ or not.

Suppose that the information of traditional products and innovative products are both dissemination in the population in the finite time horizon $[0, h]$. Assume that, at any time in the time horizon, each person in the population is in one of six possible states: potential buyers $(P)$ indicate that they do not know the information about the product, but they are easily influenced by the product information; disseminators of innovative products $(I)$ indicate that they have been influenced by the information of innovative products and have become disseminators of innovative products to disseminate innovative products; disseminators of traditional product $(T)$ indicate that they have been influenced by the information of traditional product and have become disseminators of traditional products to disseminate traditional products; vacillators (IT) indicate that they are influenced by the information of innovative products and traditional products, and the information of innovative products and traditional products is disseminated with the probability of $\alpha_{1}$ and $\alpha_{2}$ where $0 \leq \alpha_{1}, \alpha_{2} \leq 1, \quad \alpha_{1}+\alpha_{2}=1$, respectively; buyers of innovative products (UI) indicate that they have been influenced by the promotion strategies of innovative products to become buyers of innovative products; buyers of traditional products (UT) indicate that they have been influenced by the promotion strategies of traditional products to become buyers of traditional products.

Let $P_{i}(t), I_{i}(t), T_{i}(t), \mathrm{IT}_{i}(t), \mathrm{UI}_{i}(t)$, and $\mathrm{UT}_{i}(t)$ denote the probabilities of person $i(i=1,2, \ldots, N)$ being a potential buyer, a disseminator of innovative product, a disseminator of traditional product, a vacillator, a buyer of innovative products, and a buyer of traditional products at time $t$, respectively. As $\mathrm{UT}_{i}(t)=1-P_{i}(t)-I_{i}(t)-R_{i}(t)-\mathrm{IT}_{i}(t)-\mathrm{UI}_{i}(t), \quad$ the vector

$$
\mathbf{E}(t)=\left(P_{1}(t), \ldots, P_{N}(t), I_{1}(t), \ldots, I_{N}(t), T_{1}(t), \ldots, T_{N}(t), \mathrm{IT}_{1}(t), \ldots, \mathrm{IT}_{N}(t), \mathrm{UI}_{1}(t), \ldots, \mathrm{UI}_{N}(t)\right)
$$

stands for the expected state of the population at time $t$. For brevity, let

$$
\left\{\begin{array}{l}
\mathbf{P}(t)=\left(P_{1}(t), \ldots, P_{N}(t)\right), \\
\mathbf{I}(t)=\left(I_{1}(t), \ldots, I_{N}(t)\right), \\
\mathbf{T}(t)=\left(T_{1}(t), \ldots, T_{N}(t)\right), \\
\mathbf{I T}(t)=\left(\operatorname{IT}_{1}(t), \ldots, \mathrm{IT}_{N}(t)\right), \\
\mathbf{U I}(t)=\left(\mathrm{UI}_{1}(t), \ldots, \mathrm{UI}_{N}(t)\right)
\end{array}\right.
$$

Then, $\mathbf{E}(t)=(\mathbf{P}(t), \mathbf{I}(t), \mathbf{T}(t), \mathbf{I T}(t), \mathbf{U I}(t))$.

\subsection{A Mixed Dissemination Model of Traditional Product and Innovative Product. When the innovative product is in- troduced, potential buyers may not rush to adopt it. Some first-time traditional buyers may still purchase the tradi- tional product, possibly because they do not know the availability of the innovative product, or because they perceive the innovative product as unproven or lacking product support. We implement advertisement strategies for potential buyers and promotion strategies for disseminators of innovative product. When traditional product and in- novative product are disseminated on the same network, we build the following assumptions to describe the spread process of innovative products and traditional product information.}

$\left(H_{1}\right)$ Dynamic changes of potential buyers nodes: potential buyers $P_{i}$ contacting disseminators of innovative product $I_{j}$ and the former turn into disseminators of innovative product at a rate of $\sum_{j=1}^{N} \beta_{i j}^{P} I_{j}(t)$ at time $t ; \beta_{i j}^{P}$ means the average rate which is due to the influence of disseminators of innovative product $I_{j}$; potential buyers $P_{i}$ contacting disseminators of traditional product $T_{j}$ and the former turn into disseminators of traditional product at a rate of $\sum_{j=1}^{N} \gamma_{i j}^{p_{j}} T_{j}(t)$ at time $t ; \gamma_{i j}^{P}$ means the average rate which is due to the influence of disseminators of traditional product $T_{j}$; potential buyers $P_{i}$ receiving advertisement information of the innovative product turn into disseminators of innovative product $I_{i}$ with probability of $\theta_{1 i}(t)$ at time $t$; potential buyers $P_{i}$ receiving advertisement information of the traditional product turn into disseminators of traditional product $T_{i}$ with probability of $\theta_{2 i}(t)$ at time $t$.

$\left(\mathrm{H}_{2}\right)$ Dynamic changes of disseminators of innovative product node: disseminators of innovative product $I_{i}$ contacting vacillators $\mathrm{IT}_{j}$ and the former turn into vacillators at a rate of $\left(\alpha_{2} / \alpha_{1}+\alpha_{2}\right) \sum_{j=1}^{N} \beta_{i j}^{I} \mathrm{IT}_{j}(t)$ at time $t$, where vacillators disseminate two types of product information and $\beta_{i j}^{I}$ means the average rate which is due to the influence of disseminators of innovative product $I_{i}$ or vacillators $\mathrm{IT}_{j}$; disseminators of innovative product $I_{i}$ contacting disseminators of traditional product $T_{j}$ and the former turn into vacillators $\mathrm{IT}_{i}$ at a rate of $\sum_{j=1}^{N} \beta_{i j}^{I} T_{j}(t)$ at time $t$; disseminators of innovative product $I_{i}$ receiving promotion information of the 
innovative product turn into buyers of innovative products $\mathrm{UI}_{i}$ at a rate of $\beta_{1 i}(t)$ at time $t$.

$\left(\mathrm{H}_{3}\right)$ Dynamic changes of disseminators of traditional product node: disseminators of traditional product $T_{i}$ and the former contacting vacillators $\mathrm{IT}_{j}$ turn into vacillators at a rate of $\left(\alpha_{1} / \alpha_{1}+\alpha_{2}\right) \sum_{j=1}^{N} \gamma_{i j}^{T} \mathrm{IT}_{j}(t)$ at time $t$ and $\gamma_{i j}^{T}$ means the average rate which is due to the influence of disseminators of innovative product or vacillators $\mathrm{IT}_{j}$; disseminators of traditional product $T_{i}$ contacting disseminators of innovative product $I_{j}$ and the former turn into vacillators at a rate of $\sum_{j=1}^{N} \gamma_{i j}^{T} I_{j}(t)$ at time $t$; disseminators of traditional product $T_{i}$ receiving promotion information of the traditional product turn into buyers of traditional products at a rate of $\beta_{2 i}(t)$ at time $t$.

$\left(H_{4}\right)$ Dynamic changes of node $\mathrm{IT}_{j}$ : vacillators receiving promotion information of the innovative product turn into buyers of innovative products $\mathrm{UI}_{i}$ at a rate of $\beta_{1 i}(t)$ at time $t$; and those receiving promotion information of the traditional product turn into buyers of traditional products $\mathrm{UT}_{i}$ at a rate of $\beta_{2 i}(t)$ at time $t$.

Figure 1 shows this collection of assumption schematically.

Remark 1. Obviously, $\beta_{i j}^{I}=\gamma_{i j}^{P}=\beta_{i j}^{I}=\gamma_{i j}^{T}=0$, if $a_{i j}=0$.

For brevity, let

$$
\begin{aligned}
& \mathbf{B}^{P}=\left(\beta_{i j}^{P}\right)_{N \times N^{\prime}}, \\
& \mathbf{R}^{P}=\left(\gamma_{i j}^{P}\right)_{N \times N^{\prime}}, \\
& \mathbf{B}^{I}=\left(\beta_{i j}^{I}\right)_{N \times N^{\prime}}, \\
& \mathbf{R}^{T}=\left(\gamma_{i j}^{T}\right)_{N \times N} .
\end{aligned}
$$

By the total probability formula, we get the following system:

$$
\left\{\begin{array}{l}
\frac{\mathrm{d} P_{i}(t)}{\mathrm{d} t}=-P_{i}(t) \sum_{j=1}^{N} \beta_{i j}^{p} I_{j}(t)-P_{i}(t) \sum_{j=1}^{N} \gamma_{i j}^{p} T_{j}(t)-\theta_{1 i}(t) P_{i}(t)-\theta_{2 i}(t) P_{i}(t), \\
\frac{\mathrm{d} I_{i}(t)}{\mathrm{d} t}=P_{i}(t) \sum_{j=1}^{N} \beta_{i j}^{p} I_{j}(t)-I_{i}(t) \frac{\alpha_{2}}{\alpha_{1}+\alpha_{2}} \sum_{j=1}^{N} \beta_{i j}^{I} \mathrm{IT}_{j}(t)-I_{i}(t) \sum_{j=1}^{N} \beta_{i j}^{I} T_{j}(t)-\beta_{1 i}(t) I_{i}(t)+\theta_{1 i}(t) P_{i}(t), \\
\frac{\mathrm{d} T_{i}(t)}{\mathrm{d} t}=P_{i}(t) \sum_{j=1}^{N} \gamma_{i j}^{p} T_{j}(t)-T_{i}(t) \frac{\alpha_{1}}{\alpha_{1}+\alpha_{2}} \sum_{j=1}^{N} \gamma_{i j}^{T} \mathrm{IT}_{j}(t)-T_{i}(t) \sum_{j=1}^{N} \gamma_{i j}^{T} I_{j}(t)-\beta_{2 i}(t) T_{i}(t)+\theta_{2 i}(t) P_{i}(t), \\
\frac{\mathrm{dIT}(t)}{\mathrm{d} t}=I_{i}(t) \sum_{j=1}^{N}\left[\beta_{i j}^{I} T_{j}(t)+\frac{\alpha_{2}}{\alpha_{1}+\alpha_{2}} \beta_{i j}^{I} \mathrm{IT}_{j}(t)\right]+T_{i}(t) \sum_{j=1}^{N}\left[\gamma_{i j}^{T} I_{j}(t)+\frac{\alpha_{1}}{\alpha_{1}+\alpha_{2}} \gamma_{i j}^{T} \mathrm{IT}_{j}(t)\right]-\beta_{1 i}(t) \mathrm{IT}_{i}(t)-\beta_{2 i}(t) \mathrm{IT}_{i}(t), \\
\frac{\mathrm{dUI}(t)}{\mathrm{d} t}=\beta_{1 i}(t) I_{i}(t)+\beta_{1 i}(t) \mathrm{IT}_{i}(t),
\end{array}\right.
$$

where $0 \leq t \leq h, 1 \leq i \leq N$, and $\mathbf{E}(0)=\mathbf{E}_{0}$. We refer to the system as a mixed communication model of traditional product and innovative product.

\subsection{Formulating an Advertisement and Promotion Strategies.} Developing new product and accelerating the update of product are important means for enterprise to continuous operation and development. This article mainly considers the diffusion of innovative products and focuses on innovative product advertisement strategies and promotion strategies. Let $\theta_{1}$ denote the rate at which the potential buyer $P_{i}$ is affected by the advertisement of the innovative product and become disseminators of innovative product at time $t$, and $\beta_{1}$ is the rate at which $t$ disseminators of innovative product $I_{i}$ are influenced by the promotion to become buyers of innovative products. We are referring to an $\mathrm{N}$ dimensional vector-valued function $\mathbf{u}$ defined by

$$
\mathbf{u}(t)=\left(\theta_{1 i}(t), \ldots, \theta_{1 N}(t)\right), \quad 0 \leq t \leq T,
$$

as an advertisement strategy, the $N$-dimensional vectorvalued function $\mathbf{v}$ defined by

$$
\mathbf{v}(t)=\left(\beta_{1 i}(t), \ldots, \beta_{1 N}(t)\right), \quad 0 \leq t \leq T,
$$

as a promotion strategy, and the $2 \mathrm{~N}$-dimensional vectorvalued function $\mathbf{w}$ defined by

$$
\mathbf{w}(t)=\left(\theta_{1 i}(t), \cdots, \theta_{1 N}(t), \beta_{1 i}(t), \ldots, \beta_{1 N}(t)\right), \quad 0 \leq t \leq T,
$$

as an advertisement-promotion (AP) strategy. For this purpose, we assume that the admissible set of the AP strategy is

$$
W=\left\{\mathbf{w} \in[0, h]^{2 N} \mid \theta_{1 i}(t) \leq \overline{\theta_{1 i}}, \beta_{1 i}(t) \leq \overline{\beta_{1 i}}, 0 \leq t \leq h, 1 \leq i \leq N\right\},
$$




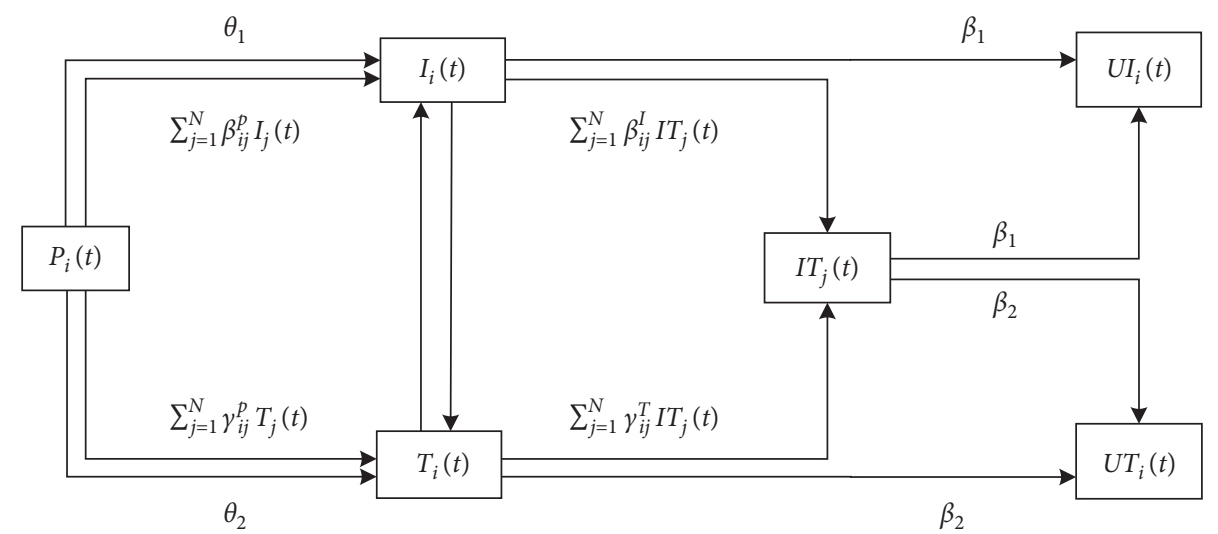

Figure 1: Diagram of traditional and innovative product information mixed spreading model.

where $L[0, h]$ stands for the set of all the Lebesgue integrable functions defined on $[0, h][30]$.

For brevity, let

$$
\overline{\mathbf{u}}=\left(\overline{\theta_{11}}, \ldots, \overline{\theta_{1 i}}\right), \overline{\mathbf{v}}=\left(\overline{\beta_{11}}, \ldots, \overline{\beta_{1 i}}\right) .
$$

Model (4) may be written in matrix notation as

$$
\left\{\begin{array}{l}
\frac{\mathrm{d} \mathbf{E}(t)}{\mathrm{d} t}=f(\mathbf{E}(t), \mathbf{w}(t)), \quad 0 \leq t \leq h, \\
\mathbf{E}(0)=\mathbf{E}_{0} .
\end{array}\right.
$$

2.4. Measuring the Influence of Innovative Products. We use the revenue from innovative products to express the influence of innovative products. The benefits of influencing innovative products are composed of four parts: the benefits of indirect influence from the disseminators of innovative products, the benefits of buyers of innovative products, the cost of implementing advertising strategies, and the cost of implementing promotional strategies.

Firstly, assume the indirect income per unit time caused by the disseminators of innovative product $I_{i}$ is $a_{i}$ units (for example, US dollars), which is a constant. Then the expected indirect income in the time horizon $[0, h]$ caused by the innovative product information is

$$
J_{1}(\mathbf{w})=\int_{0}^{h} \sum_{i=1}^{N} a_{i} I_{i}(t) \mathrm{d} t
$$

units. We use this quantity to measure the indirect income caused by information of innovative product.
Secondly, assume the direct income per unit time caused by the buyers of innovative products $\mathrm{UI}_{i}$ is $b_{i}$ units, which is a constant. Then the expected direct income in the time horizon $[0, h]$ is

$$
J_{2}(\mathbf{w})=\int_{0}^{h} \sum_{i=1}^{N} b_{i} \mathrm{UI}_{i}(t) \mathrm{d} t
$$

We use this quantity to measure direct income caused by buyers of innovative products.

Thirdly, assume the cost per unit time for converting potential buyers $P_{i}$ at $\theta_{1}$ rate is $c_{i} \theta_{1}$ units, which is a constant. Then the expected cost for implementing the advertisement strategies $\mathbf{u}$ is

$$
J_{3}(\mathbf{w})=\int_{0}^{h} \sum_{i=1}^{N} c_{i} \theta_{1 i}(t) P_{i}(t) \mathrm{d} t .
$$

We use this quantity to measure the cost for implementing the advertisement strategies $u$.

Finally, assume the cost per unit time for converting disseminators of innovative product $I_{i}$ or vacillators $\mathrm{IT}_{i}$ at the rate of $\beta_{1}$ is $\beta_{1} d_{i}$ units, which is a constant. Then the expected cost for implementing the promotion strategies $\mathbf{v}$ is

$$
J_{4}(\mathbf{w})=\int_{0}^{h} \sum_{i=1}^{N} d_{i} \beta_{1 i}(t)\left(I_{i}(t)+\mathrm{UI}_{i}(t)\right) \mathrm{d} t .
$$

We use this quantity to measure the cost for implementing the promotion strategies $\mathbf{v}$.

Combined with the above discussion, the expected revenue of innovative products is

$$
\begin{aligned}
J(\mathbf{w}) & =J_{1}(\mathbf{w})+J_{2}(\mathbf{w})-J_{3}(\mathbf{w})-J_{4}(\mathbf{w}) \\
& =\int_{0}^{h} \sum_{i=1}^{N} a_{i} I_{i}(t) \mathrm{d} t+\int_{0}^{h} \sum_{i=1}^{N} b_{i} \mathrm{UI}_{i}(t) \mathrm{d} t-\int_{0}^{h} \sum_{i=1}^{N} c_{i} \theta_{1 i}(t) P_{i}(t) \mathrm{d} t-\int_{0}^{h} \sum_{i=1}^{N} d_{i} \beta_{1 i}(t)\left(I_{i}(t)+\mathrm{IT}_{i}(t)\right) \mathrm{d} t .
\end{aligned}
$$


We use this quantity to measure the influence of the innovative products.

For brevity, let

$$
\begin{aligned}
& \mathbf{a}=\left(a_{1}, \ldots, a_{N}\right), \\
& \mathbf{b}=\left(b_{1}, \ldots, b_{N}\right), \\
& \mathbf{c}=\left(c_{1}, \ldots, c_{N}\right), \\
& \mathbf{d}=\left(d_{1}, \ldots, d_{N}\right) .
\end{aligned}
$$

2.5. Modeling the Advertisement and Promotion Strategies. Based on the previous discussions, the advertisement and promotion (AP) strategies are modeled as the following optimal control problem:

$$
\begin{aligned}
& \operatorname{Max}_{\mathbf{w} \in W} J(\mathbf{w})=\int_{0}^{h} F(\mathbf{E}(t), \mathbf{w}(t)) \mathrm{d} t \\
& \text { Subject to }\left\{\begin{array}{l}
\frac{\mathbf{d E}(t)}{\mathbf{d} t}=f(\mathbf{E}(t), \mathbf{w}(t)), \quad 0 \leq t \leq h, \\
\mathbf{E}(0)=\mathbf{x}_{0},
\end{array}\right.
\end{aligned}
$$

where

$$
\begin{aligned}
F(\mathbf{E}(t), \mathbf{w}(t))= & \int_{0}^{h} \sum_{i=1}^{N} a_{i} I_{i}(t) \mathrm{d} t+\int_{0}^{h} \sum_{i=1}^{N} b_{i} \mathrm{UI}_{i}(t) \mathrm{d} t \\
& -\int_{0}^{h} \sum_{i=1}^{N} c_{i} \theta_{1 i}(t) P_{i}(t) \mathrm{d} t-\int_{0}^{h} \sum_{i=1}^{N} d_{i} \beta_{1 i} \\
& (t)\left(I_{i}(t)+\operatorname{IT}_{i}(t)\right) \mathrm{d} t .
\end{aligned}
$$

We refer to the optimal control problem as the advertisement-promotion (AP) strategies. In this model, the control stands for the AP strategies, the objective functional stands for the influence of the innovative products, and the optimal control stands for the optimal AP strategies.

The AP model (17) can be characterized as the following 12-tuple:

$$
\Gamma=\left(h, \overline{\mathbf{u}}, \overline{\mathbf{v}}, \mathbf{B}^{P}, \mathbf{R}^{P}, \mathbf{B}^{I}, \mathbf{R}^{T}, \mathbf{a}, \mathbf{b}, \mathbf{c}, \mathbf{d}, \mathbf{E}_{0}\right) .
$$

\section{A Method for Solving the AP Strategies}

In the previous section, we modeled the AP strategies as an optimal control problem, i.e., the AP strategy (17) or (19). In this section, we derive a method for solving the AP strategy. First, we show that the AP strategy is solvable by proving that it admits an optimal control. Second, we give a necessary condition for the AP strategy. On this basis, we present the optimality system for solving the AP strategy.

3.1. The Existence of an Optimal Control. To show the AP strategy admits an optimal control, we need the following lemma [31].
Lemma 1. The AP strategy (19) has an optimal control if the following five conditions hold simultaneously:

(C1) $W$ is bounded and convex.

(C2) There is $\mathbf{w} \in W$ such that the corresponding state evolution model (9) is solvable.

(C3) $f(\mathbf{E}, \mathbf{w})$ is bounded by a linear function in $\mathbf{E}$.

(C4) $F(\mathbf{E}, \mathbf{w})$ is convex on $W$.

(C5) $F(\mathbf{E}, \mathbf{w}) \geq d_{1}\|\mathbf{w}\|_{2}^{\rho}+d_{2}$ for some $\rho>1, d_{1}>0$ and $d_{2}$.

We are ready to show the following result.

Theorem 1. The AP strategy (19) admits an optimal control.

Proof. Let $\mathbf{w}=\left(\theta_{11}, \ldots, \theta_{1 N}, \beta_{11}, \ldots, \beta_{1 N}\right)$ be a limit point of $W$. Then there exists a sequence of points of $W$,

$$
\mathbf{w}^{(n)}=\left(\theta_{11}^{(n)}, \ldots, \theta_{1 N}^{(n)}, \beta_{11}^{(n)}, \ldots, \beta_{1 N}^{(n)}\right), \quad n=1,2, \ldots,
$$

which approaches $\mathbf{w}$. As $L[0, h]^{2 N}$ is complete, we have $\mathbf{w} \in L[0, h]^{2 N}$. So, $\mathbf{w} \in W$ follows from the observation that

$$
\begin{aligned}
\theta_{1 i}(t) & =\lim _{n \longrightarrow \infty} \theta_{1 i}^{(n)}(t) \leq \overline{\theta_{1 i}}, \\
\beta_{1 i}(t) & =\lim _{n \longrightarrow \infty} \beta_{1 i}^{(n)}(t) \leq \overline{\beta_{1 i}}, \\
0 & \leq t \leq h, 1 \leq i \leq N .
\end{aligned}
$$

Hence, $W$ is closed. Let $\mathbf{w}^{(1)}, \mathbf{w}^{(2)} \in W, 0<\eta<1$. As $L[0, h]^{2 N}$ is a real vector space, we have $(1-\eta) \mathbf{w}^{(1)}+$ $\eta \mathbf{w}^{(2)} \in L[0, h]^{2 N}$. So, $(1-\eta) \mathbf{w}^{(1)}+\eta \mathbf{w}^{(2)} \in W$ follows from the observation that

$$
\begin{aligned}
& (1-\eta) \theta_{1 i}^{(1)}(t)+\eta \theta_{1 i}^{(2)}(t) \leq \overline{\theta_{1 i}},(1-\eta) \beta_{1 i}^{(1)}(t)+\eta \beta_{1 i}^{(2)}(t) \\
& \leq \overline{\beta_{1 i}}, \quad 0 \leq t \leq h, 1 \leq i \leq N .
\end{aligned}
$$

Hence, $W$ is convex. The first condition in Lemma 1 is proven. Let $\overline{\mathbf{w}}(t) \equiv\left(\overline{\theta_{11}}, \ldots, \overline{\theta_{1 N}}, \overline{\beta_{11}}, \ldots, \overline{\beta_{1 N}}\right)$. Then $\overline{\mathbf{w}} \in W$. As $f(\mathbf{E}, \overline{\mathbf{w}})$ is continuously differentiable, it follows by the continuation theorem for differential systems that the corresponding state evolution state is solvable. The second condition is proven. The third condition follows from the boundedness of $P_{i}, I_{i}, T_{i}, \mathrm{IT}_{i}, \mathrm{UI}_{i}$, and $\mathbf{w}$, and the fourth condition follows from that $F$ is linear in $\mathbf{w}$ and hence is convex. The fifth condition follows from the observation that

$$
\begin{aligned}
F(\mathbf{E}, \mathbf{w}) & \geq 0 \geq \sum_{i=1}^{N}\left(\theta_{1 i}^{2}+\beta_{1 i}^{2}\right)-\sum_{i=1}^{N}\left({\overline{\theta_{1 i}}}^{2}+{\overline{\beta_{1 i}}}^{2}\right) \\
& =\|\mathbf{w}\|_{2}^{2}-\sum_{i=1}^{N}\left({\overline{\theta_{1 i}}}^{2}+{\overline{\beta_{1 i}}}^{2}\right) .
\end{aligned}
$$

By Lemma 1, the proposition holds.

3.2. A Necessary Condition for the Optimal Control. The Hamiltonian of the AP strategy (19) is 


$$
\begin{aligned}
& H(\mathbf{E}(t), \mathbf{w}(t), \mathbf{z}(t))=\sum_{i=1}^{N} a_{i} I_{i}(t)+\sum_{i=1}^{N} b_{i} \mathrm{UI}_{i}(t)-\sum_{i=1}^{N} c_{i} \theta_{1 i}(t) P_{i}(t)-\sum_{i=1}^{N} d_{i} \beta_{1 i}(t)\left(I_{i}(t)+\mathrm{IT}_{i}(t)\right) \\
& +\sum_{i=1}^{N} \lambda_{1 i}(t)\left[-P_{i}(t) \sum_{j=1}^{N} \beta_{i j}^{p} I_{j}(t)-P_{i}(t) \sum_{j=1}^{N} \gamma_{i j}^{p} T_{j}(t)-\theta_{1 i}(t) P_{i}(t)-\theta_{2 i}(t) P_{i}(t)\right] \\
& +\sum_{i=1}^{N} \lambda_{2 i}(t)\left[P_{i}(t) \sum_{j=1}^{N} \beta_{i j}^{p} I_{j}(t)-\frac{\alpha_{2}}{\alpha_{1}+\alpha_{2}} I_{i}(t) \sum_{j=1}^{N} \beta_{i j}^{I} \mathrm{IT}_{j}(t)-I_{i}(t) \sum_{j=1}^{N} \beta_{i j}^{I} T_{j}(t)-\beta_{1 i}(t) I_{i}(t)+\theta_{1 i}(t) P_{i}(t)\right] \\
& +\sum_{i=1}^{N} \lambda_{3 i}(t)\left[P_{i}(t) \sum_{j=1}^{N} \gamma_{i j}^{p_{j}} T_{j}(t)-\frac{\alpha_{1}}{\alpha_{1}+\alpha_{2}} T_{i}(t) \sum_{j=1}^{N} \gamma_{i j}^{T} \mathrm{IT}_{j}(t)-T_{i}(t) \sum_{j=1}^{N} \gamma_{i j}^{T} I_{j}(t)-\beta_{2 i}(t) T_{i}(t)+\theta_{2 i}(t) P_{i}(t)\right] \\
& +\sum_{i=1}^{N} \lambda_{4 i}(t)\left[I_{i}(t) \sum_{j=1}^{N}\left[\beta_{i j}^{I} T_{j}(t)+\frac{\alpha_{2}}{\alpha_{1}+\alpha_{2}} \beta_{i j}^{I} \operatorname{IT}_{j}(t)\right]-\beta_{1 i}(t) \operatorname{IT}_{i}(t)-\beta_{2 i}(t) \operatorname{IT}_{i}(t)\right. \\
& \left.+T_{i}(t) \sum_{j=1}^{N}\left[\gamma_{i j}^{T} I_{j}(t)+\frac{\alpha_{1}}{\alpha_{1}+\alpha_{2}} \gamma_{i j}^{T} \mathrm{IT}_{j}(t)\right]\right] \\
& +\sum_{i=1}^{N} \lambda_{5 i}(t)\left[\beta_{1 i}(t) I_{i}(t)+\beta_{1 i}(t) I T_{i}(t)\right]
\end{aligned}
$$

where $\quad \mathbf{z}(t)=\left(\lambda_{11}(t), \ldots, \lambda_{1 N}(t), \lambda_{21}(t), \ldots, \lambda_{2 N}(t), \lambda_{31}\right.$ $\left.(t), \ldots, \lambda_{3 N}(t), \lambda_{41}(t), \ldots, \lambda_{4 N}(t), \lambda_{51}(t), \ldots, \lambda_{5 N}(t)\right)$ is the adjoint.

We give a necessary condition for the optimal control of the AP strategy as follows.
Theorem 2. Suppose $\mathbf{w}$ is an optimal control of the AP strategy (24), and $\mathbf{E}$ is the solution to the corresponding model (10). Then, there exists $\mathbf{z}$ such that

$$
\left\{\begin{array}{l}
\frac{\mathrm{d} \lambda_{1 i}(t)}{\mathrm{d} t}=c_{i} \theta_{1 i}(t)+\left[\lambda_{1 i}(t)-\lambda_{2 i}(t)\right]\left[\theta_{1 i}(t)+\sum_{j=1}^{N} \beta_{i j}^{P} I_{j}(t)\right]+\left[\lambda_{1 i}(t)-\lambda_{3 i}(t)\right]\left[\theta_{2 i}(t)+\sum_{j=1}^{N} \gamma_{i j}^{P} T_{j}(t)\right] \\
\frac{\mathrm{d} \lambda_{2 i}(t)}{\mathrm{d} t}=-a_{i}+d_{i} \beta_{1 i}+\sum_{i=1}^{N}\left[\lambda_{1 i}(t)-\lambda_{2 i}(t)\right] P_{i}(t) \beta_{i j}^{P}+\sum_{i=1}^{N}\left[\lambda_{3 i}(t)-\lambda_{4 i}(t)\right] T_{i}(t) \gamma_{i j}^{T}+\left[\lambda_{2 i}(t)-\lambda_{4 i}(t)\right] \sum_{j=1}^{N} \beta_{i j}^{I}\left[\frac{\alpha_{2}}{\alpha_{1}+\alpha_{2}} I T_{j}(t)+T_{j}(t)\right]+\beta_{1 i}(t)\left[\lambda_{2 i}(t)-\lambda_{5 i}(t)\right] \\
\frac{\mathrm{d} \lambda_{3 i}(t)}{\mathrm{d} t}=\sum_{i=1}^{N}\left[\lambda_{1 i}(t)-\lambda_{3 i}(t)\right] P_{i}(t) \gamma_{i j}^{P}+\sum_{i=1}^{N}\left[\lambda_{2 i}(t)-\lambda_{4 i}(t)\right] I_{i}(t) \beta_{i j}^{I}+\lambda_{3 i}(t) \beta_{2 i}(t)+\left[\lambda_{3 i}(t)-\lambda_{4 i}(t)\right] \sum_{j=1}^{N} \frac{\alpha_{1}}{\alpha_{1}+\alpha_{2}} \gamma_{i j}^{T} \mathrm{IT} \mathrm{T}_{j}+\left[\lambda_{3 i}(t)-\lambda_{4 i}(t)\right] \sum_{j=1}^{N} \gamma_{i j}^{T} I_{j}, \\
\frac{\mathrm{d} \lambda_{4 i}(t)}{\mathrm{d} t}=d_{i} \beta_{1 i}+\sum_{i=1}^{N}\left[\lambda_{2 i}(t)-\lambda_{4 i}(t)\right] I_{i}(t) \frac{\alpha_{2}}{\alpha_{1}+\alpha_{2}} \beta_{i j}^{I}+\sum_{i=1}^{N}\left[\lambda_{3 i}(t)-\lambda_{4 i}(t)\right] T_{i}(t) \frac{\alpha_{1}}{\alpha_{1}+\alpha_{2}} \gamma_{i j}^{T}+\beta_{1 i}(t)\left[\lambda_{4 i}(t)-\lambda_{5 i}(t)\right]-\lambda_{4 i}(t) \beta_{2 i}(t) \\
\frac{\mathrm{d} \lambda_{5 i}(t)}{\mathrm{d} t}=b_{i},
\end{array}\right.
$$

where $0 \leq t \leq h, \quad 1 \leq i \leq N$, and $\mathbf{z}(h)=\mathbf{0}$. Moreover, 


$$
\begin{aligned}
& \theta_{1 i}=\left\{\begin{array}{l}
\underline{\underline{\theta_{1 i}}} c_{i}+\lambda_{1 i}(t)<\lambda_{2 i}(t), P_{i}(t)>0, \\
\overline{\theta_{1 i}} c_{i}+\lambda_{1 i}(t)>\lambda_{2 i}(t), P_{i}(t)>0, \\
0 \leq t \leq h, 1 \leq i \leq N,
\end{array}\right. \\
& \beta_{1 i}=\left\{\begin{array}{l}
\frac{\beta_{1 i}}{\overline{\beta_{1 i}}} d_{i}+\lambda_{2 i}(t)<\lambda_{2 i}(t)>\lambda_{5 i}(t) \text { and } d_{i}+\lambda_{4 i}(t)<\lambda_{5 i}(t), \lambda_{4 i}(t)>\lambda_{5 i}(t), I_{i}(t)>0, \\
0 \leq t \leq h, 1 \leq i \leq N .
\end{array}\right.
\end{aligned}
$$

Proof. It follows from the Pontryagin maximum principle [31] that there exists $\mathbf{z}$ such that

$$
\left\{\begin{array}{l}
\frac{\mathrm{d} \lambda_{1 i}(t)}{\mathrm{d} t}=-\frac{\partial H(\mathbf{E}(t), \mathbf{w}(t), \mathbf{z}(t))}{\partial P_{i}}, \\
\frac{\mathrm{d} \lambda_{2 i}(t)}{\mathrm{d} t}=-\frac{\partial H(\mathbf{E}(t), \mathbf{w}(t), \mathbf{z}(t))}{\partial I_{i}} \\
\frac{\mathrm{d} \lambda_{3 i}(t)}{\mathrm{d} t}=-\frac{\partial H(\mathbf{E}(t), \mathbf{w}(t), \mathbf{z}(t))}{\partial T_{i}}, \\
\frac{\mathrm{d} \lambda_{4 i}(t)}{\mathrm{d} t}=-\frac{\partial H(\mathbf{E}(t), \mathbf{w}(t), \mathbf{z}(t))}{\partial \mathrm{IT}} \\
\frac{\mathrm{d} \lambda_{5 i}(t)}{\mathrm{d} t}=-\frac{\partial H(\mathbf{E}(t), \mathbf{w}(t), \mathbf{z}(t))}{\partial \mathrm{UI}} \\
0 \leq t \leq h, 1 \leq i \leq N .
\end{array}\right.
$$

Equation (24) follows by direct calculations. As the terminal cost is unspecified, and the final state is free, the transversality condition $\mathbf{z}(h)=\mathbf{0}$ holds. Again by the Pontryagin maximum principle [31], we have

$$
\begin{aligned}
& H(\mathbf{E}(t), \mathbf{w}(t), \mathbf{z}(t))=\underset{\widetilde{W} \in \omega}{\arg \max } H(\mathbf{E}(t), \widetilde{\mathbf{w}}(t), \mathbf{z}(t)), \quad 0 \leq t \leq h . \\
& \begin{array}{c}
\text { Equations } \\
\text { calculations. }
\end{array}
\end{aligned}
$$

3.3. The Optimality System for the APStrategies. Based on the above discussions, (10) and (25)-(27) plus $\mathbf{z}(h)=\mathbf{0}$ constitute the optimality system for the AP strategy (19). By using the well-known forward-backward Euler scheme to the optimality system, we get an optimal control of the model, which stands for an optimal AP strategy.

Remark 2. It follows from Theorem 2 that every component of the optimal control of the AP strategy is bang-bang. In practice, this kind of optimal control is easily implementable.

\section{The Numerical Simulation}

4.1. Without Control in the Diffusion Process. In order to further study the influence of advertisement and promotion strategies on the system, the following simulation analysis is carried out in this chapter.

When an innovative product is put on the market, suppose there are 10000 people in the supply chain network, and the initial number of people in each state and parameters are set as follows:

$$
\begin{aligned}
P(0) & =8600, I(0)=200, T(0)=1000, \mathrm{IT}(0)=200, \mathrm{UI}(0)=0, \mathrm{UT}(0)=0, \\
\mathbf{B}^{P} & =0.00002, \mathbf{R}^{P}=0.000035, \mathbf{B}^{I}=0.000035, \mathbf{R}^{P}=0.00002, \theta_{2}=0.1, \beta_{2}=0.2 .
\end{aligned}
$$

Figure 2 shows the time evolution of the six states of $P \sim$ UT with $\theta_{1}$. With other parameters unchanged, the more $\theta_{1}$ increases, the more disseminators of innovative product $I$, vacillators IT, and buyers of innovative products UI increase. As companies increase the advertisement intensity of innovative products, the probability of potential buyer $P$ acquiring innovative product advertisement information increases. The probability of potential buyer $P$ changing into disseminators of traditional product disseminator of innovative product $I$ increases, and the probability of disseminators of traditional product $T$ changing into vacillators IT increases. As a result, the number of buyers of innovative products UI has increased significantly. The number of buyers of vacillators IT and buyers of innovative products UI reaches a maximum in a shorter time. It can be concluded that advertisement has a more obvious effect on improving the market influence of innovative products.
Figure 3 shows the changes of four states of $I$, IT, UI, and UT with time. In the case of other parameters unchanged, the more $\beta_{1}$ increases, the more the number of buyers of innovative products UI increases, and the number of vacillators IT and buyers of traditional products UT decreases. As the company increases its promotional strategy, the possibility of disseminators of innovative product $I$ and vacillators IT changing to buyers of innovative products UI increases. Similarly, the possibility of disseminators of innovative product $I$ becoming vacillators IT is reduced. The possibility of vacillators IT becoming buyers of traditional products UT is reduced. The number of buyers of innovative products UI has increased significantly and reached a maximum in a relatively short period of time. It can be concluded that promotion has an obvious effect on improving the market influence of innovative products. 

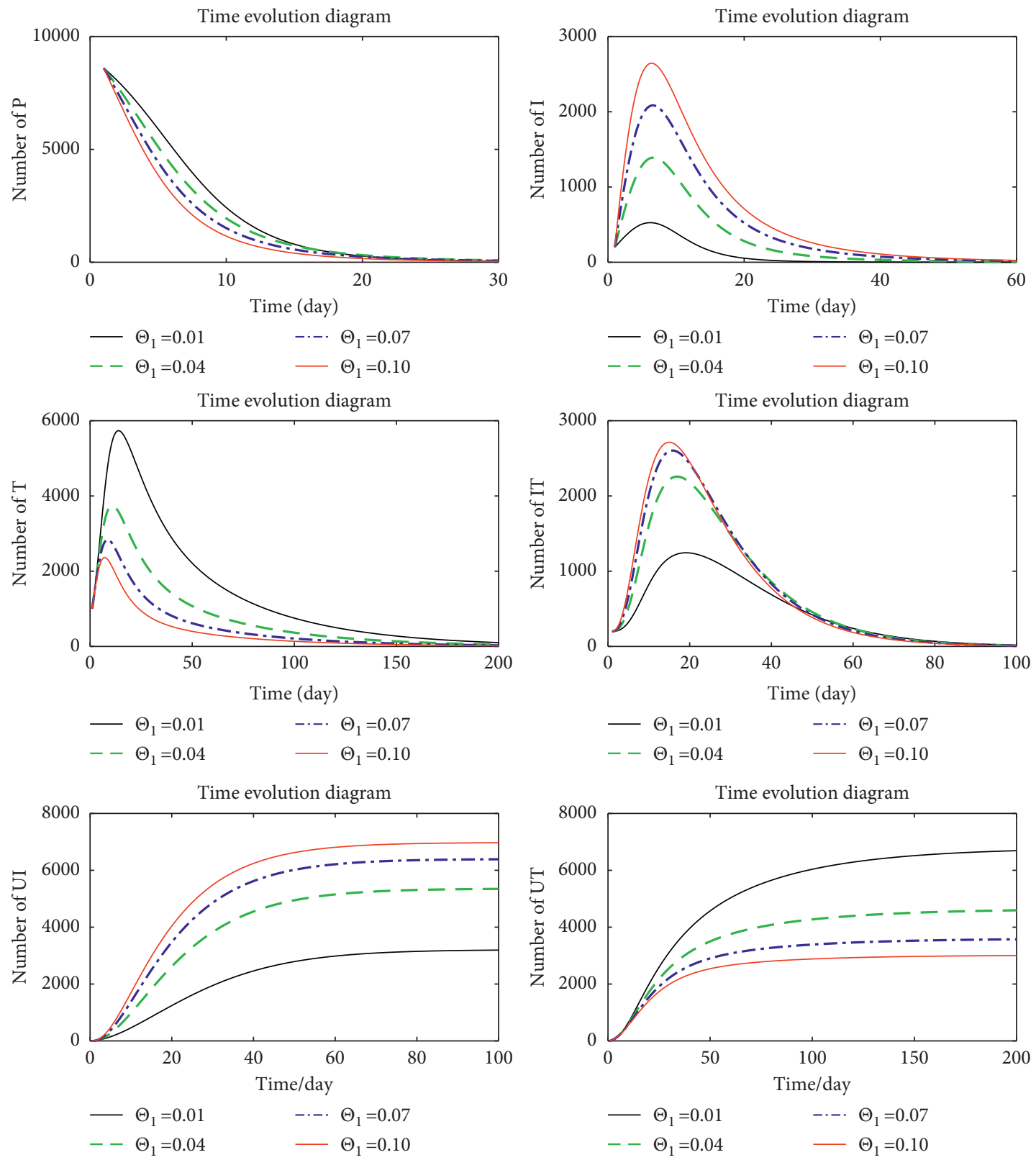

Figure 2: Time evolution graph of the number of nodes changing with a rate of $\theta_{1}$.

4.2. Optimal AP Strategies in the Diffusion Process. When all parameters are set as constants without considering optimal control, $\theta_{1}$ and $\beta_{1}$ have a great influence on $J$. Below we discuss optimal AP strategies in the diffusion process; the parameters $\theta_{1}(t)$ and $\beta_{1}(t)$ show dynamic changes with time $t$ on $J$.

Figure 4 shows the number of people with control (solid line) and no control (dashed line) in $I, T$, IT, and UT. In with control situation, $\theta_{1}$ and $\beta_{1}$ are dynamically changing and other parameters are constant. Obviously, under the control (solid line), the numbers of disseminators of traditional product $T$ and buyers of traditional products UT decrease, and disseminators of traditional product $T$ reach the peak faster because companies implement advertisement strategies to increase the probability of potential buyers $P$ transforming into disseminators of innovative product $I$; companies implement promotion strategies to increase the probability that disseminators of innovative product $I$ and vacillators IT will transform into buyers of innovative products IT, so the number of buyers of traditional products UT declines. Conversely, when there is no control (dashed line), the relative probability of potential buyers $P$ changing to disseminators of traditional product $T$ increases, and the relative probability of converting disseminators of traditional product $T$ and vacillators IT to buyers of traditional products UT increases, so the number of buyers of traditional products UT rose. In short, companies implementing advertisement and product promotion strategies have significantly increased the market influence of innovative products. 

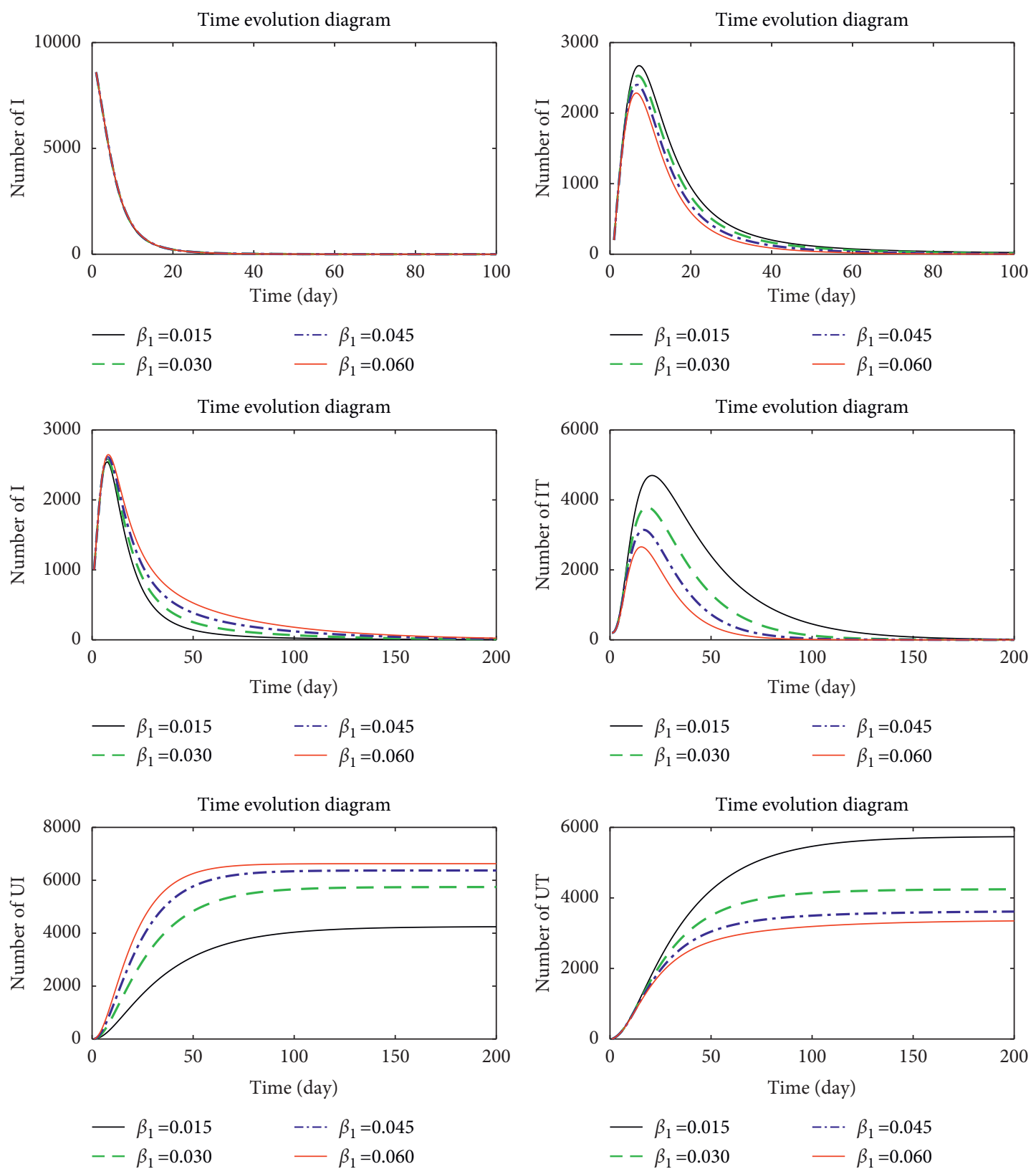

FIgURE 3: Time evolution graph of the number of nodes changing with a rate of $\beta_{1}$.

Inspired by the forward-backward sweep method for solving differential systems [32], in Algorithm 1 as in Table 1, we describe an algorithm (OS-FBS algorithm, where OS-FBS stands for optimality system forward-backward sweep) for solving the optimality system of an AP strategy. In all of the following experiments, we set $\varepsilon=10^{-5}, K=10^{3}$.

Use OS-FBS algorithm to find the maximum value, the results of multiple distribution schemes under the control of advertisement and promotion information, and also include an uncontrolled result. Except for $\theta_{1}$ and $\beta_{1}$, other simulation parameter values are unchanged. The overall results are shown in Table 1 as below.
Figure 5 shows the corresponding economic benefits of different schemes, including the economic benefits of the 37 allocation schemes under control and the economic benefits of uncontrolled. It can be clearly seen from the figure that the controlled economic benefits are obviously more than the uncontrolled economic benefits $(J(\mathbf{w})=6374)$, among which the maximum distribution scheme $\left(\theta_{1}=0.6\right.$, $\left.\beta_{1}=0.1\right)$ and the economic benefits are $(J(\mathbf{w})=471467)$.

In short, optimal AP strategies can increase the influence of innovative products on the market, thereby increasing corporate earnings. In this case, the advertisement strategy investment is 0.6 , and the promotion strategy investment is 


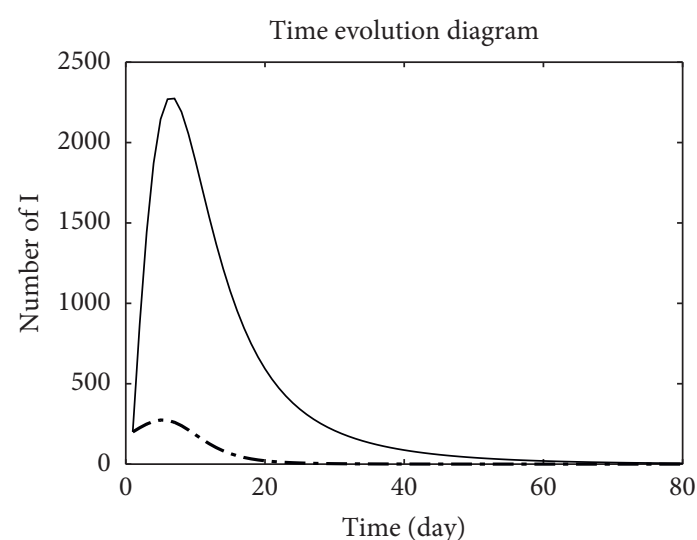

- Controlled I

-.-. Without control I

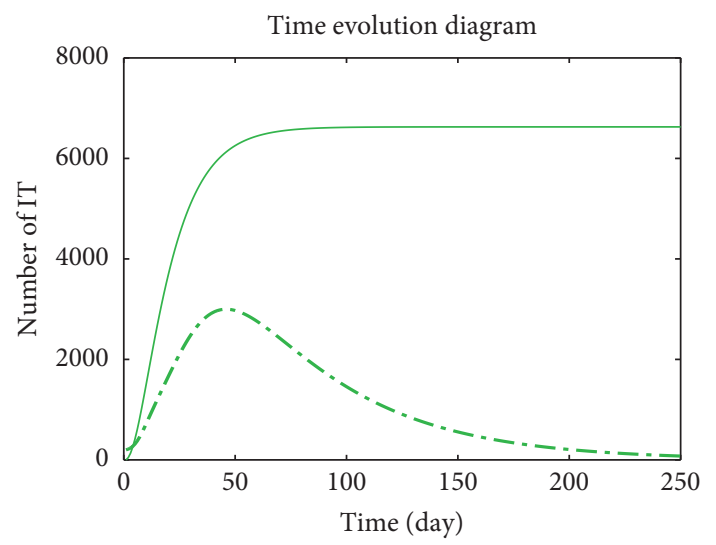

- Controlled IT

-... Without control IT

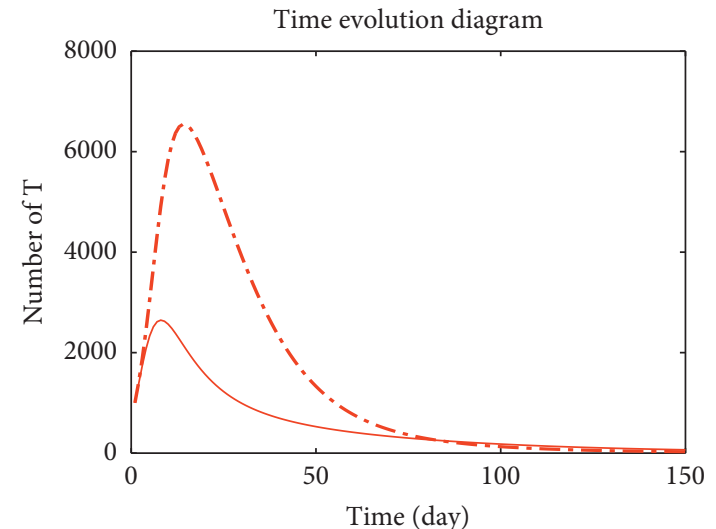

- Controlled T

-.-. Without control T

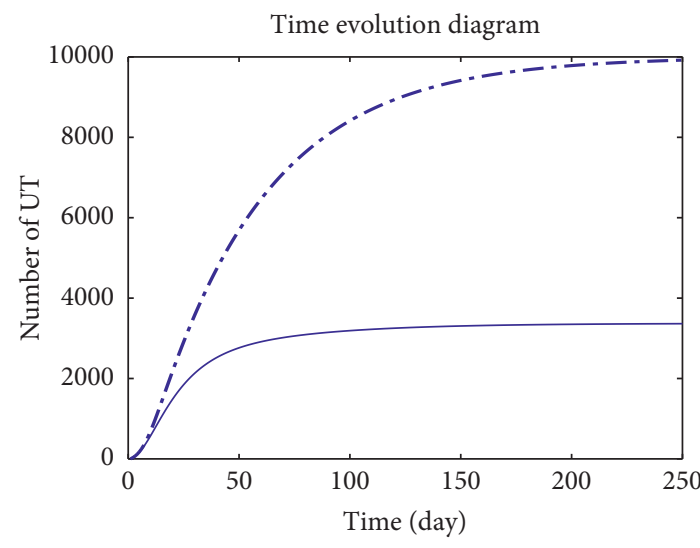

Controlled UT

-.-. Without control UT

Figure 4: Time evolution graph of the number of nodes changing with and without control.

Input: a CPD game $G=\left(G, a, b, c, d, h, R, S, \bar{\theta}, \bar{\beta}, E_{0}\right)$, a convergence error $\varepsilon$, an upper bound $K$ on the number of iteration steps. Output: a strategy-pair $(\theta, \beta)$

(1) //initialize;//

(2) $k:=0$;

(3) $\theta^{(0)}=0 ; \beta^{(0)}=0$

(4) b//generate the strategy-pair through iterations;//

(5) repeat

(6) $k=k+1$;

(7) use (1) with $\theta=\theta^{(k-1)}, \beta=\beta^{(k-1)}$, and $E(0)=E_{0}$ to forwards calculate $E$;

(8) $E^{(k)}:=E$;

(9) use (6) with $\theta=\theta^{(k-1)}, \beta=\beta^{(k-1)}$, and $E=E^{(k)}$, and $\lambda(h)=\mu(h)=0$ to backwards calculate $\lambda$ and $\mu$;

(10) $\quad \lambda^{(k)}:=\lambda ; \mu^{(k)}:=\mu$

(11) use (7)-(10) with $E=E^{(k)}, \lambda=\lambda^{(k)}$, and $\mu=\mu^{(k)}$ to calculate $\theta$ and $\beta$;

(12) $\theta^{(k)}=\theta ; \beta^{(k)}=\beta$;

(13) until $\left\|\theta^{(k)}-\theta^{(k-1)}\right\|+\left\|\beta^{(k)}-\beta^{(k-1)}\right\|<\varepsilon$ or $k>K$;

(14) return $\left(\theta^{(k)}, \beta^{(k)}\right)$

Algorithm 1: OS-FBS. 
TABLE 1: Scheme distribution and economic benefits under control.

\begin{tabular}{|c|c|}
\hline Allocation & Income \\
\hline $0.6,0.1$ & 471467 \\
\hline $0.6,0.3$ & 425431 \\
\hline $0.2,0.1$ & 430197 \\
\hline $0.5,0.3$ & 423956 \\
\hline $0.6,0.5$ & 404657 \\
\hline $0.4,0.3$ & 419345 \\
\hline $0.6,0.6$ & 398337 \\
\hline $0.5,0.5$ & 402533 \\
\hline $0.6,0.8$ & 389655 \\
\hline $0.6,0.9$ & 386549 \\
\hline $0.5,0.6$ & 395948 \\
\hline $0.5,0.1$ & 469460 \\
\hline Without control & 6374 \\
\hline $0.1,0.9$ & 249337 \\
\hline $0.1,0.7$ & 259249 \\
\hline $0.2,0.9$ & 330986 \\
\hline $0.1,0.6$ & 266207 \\
\hline $0.3,0.9$ & 362615 \\
\hline $0.2,0.8$ & 335236 \\
\hline $0.4,0.9$ & 376814 \\
\hline $0.5,0.9$ & 383570 \\
\hline $0.3,0.8$ & 366429 \\
\hline $0.4,0.8$ & 380315 \\
\hline $0.5,0.8$ & 386846 \\
\hline $0.4,0.1$ & 464476 \\
\hline $0.2,0.6$ & 346969 \\
\hline $0.1,0.4$ & 287737 \\
\hline $0.3,0.6$ & 376940 \\
\hline $0.4,0.6$ & 389990 \\
\hline $0.3,0.4$ & 394507 \\
\hline $0.4,0.4$ & 406277 \\
\hline $0.2,0.3$ & 382151 \\
\hline $0.1,0.2$ & 331480 \\
\hline $0.3,0.3$ & 408450 \\
\hline $0.2,0.2$ & 404209 \\
\hline $0.1,0.1$ & 363797 \\
\hline $0.3,0.1$ & 453859 \\
\hline
\end{tabular}

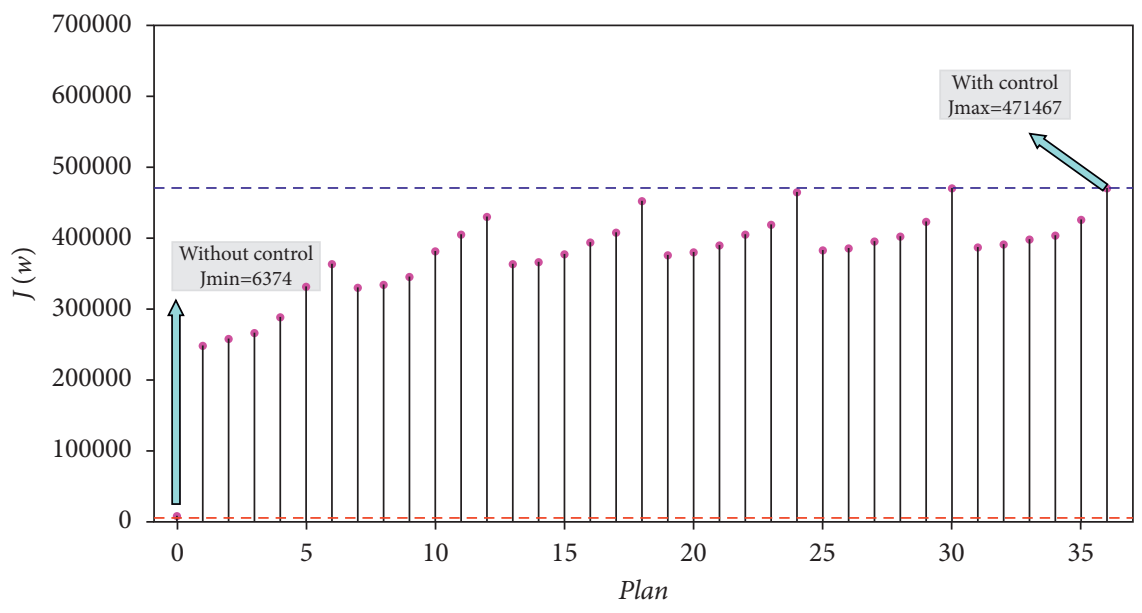

FIGURE 5: Economic benefits corresponding to different scenarios. 
0.1, which further maximizes the economic benefits, which also represents the effectiveness of the control provided by the enterprise.

\section{Conclusions}

Based on the competition between traditional products and alternative innovative products, after considering advertising and promotion strategies to expand the influence factor of innovative products, a diffusion model of innovative products has been proposed. In order to further expand the influence of innovative products, we propose the optimal control problem of innovative products. Finally, the Pontryagin maximum principle has solved the optimal solution. We have simulated the diffusion process of innovative products through simulation. The analysis results of model have shown that, in the process of competition between innovative products and traditional products, the advertisement strategy of innovative products has promoted the information dissemination of innovative products, and the promotion strategy of innovative products has further increased the market share of innovative products. The research in this paper has provided a reference for some companies to implement market strategies.

We appropriately acknowledge the potential limitations of this study. We use fixed parameters to simulate the diffusion process of innovative products. Collecting real data to calculate the real transformation probability function or transformation value is the next research direction. Research on the diffusion of innovative products in a uniform network has certain limitations, and further research on the diffusion of products in a heterogeneous network is needed. In the process of implementing market strategies for innovative products, the market strategies of traditional products cannot remain unchanged. Moving in this direction, there are some research topics worthy of further study. Consider the influence of cooperative and noncooperative games between enterprises in the product diffusion model.

\section{Data Availability}

The data used to support the findings of this study are included within the article.

\section{Conflicts of Interest}

The authors declare that they have no conflicts of interest.

\section{Acknowledgments}

This work was partially supported by the Program for Professor of Special Appointment (Eastern Scholar) at Shanghai Institutions of Higher Learning, the National Natural Science Foundation of China (71774111, 61702331, and 71871144), and Science and Technology Development Program of University of Shanghai for Science and Technology (2020KJFZ0460).

\section{References}

[1] F. M. Bass, "A new product growth for model consumer durables," Management Science, vol. 15, no. 5, pp. 215-227, 1969.

[2] W. A. Kamakura and S. K. Ealasubramanian, "Long-term forecasting with innovation diffusion models: the impact of replacement purchases," Journal of Forecasting, vol. 6, no. 1, pp. 1-19, 1987.

[3] M. U. Kalwani and A. J. Silk, "Structure of repeat buying for new packaged goods," Journal of Marketing Research, vol. 17, no. 3, pp. 316-322, 1980.

[4] V. Fanelli and L. Maddalena, "A time delay model for the diffusion of a new technology," Nonlinear Analysis: Real World Applications, vol. 13, no. 2, pp. 643-649, 2012.

[5] N. I. Shaikh, A. Rangaswamy, and A. Balakrishnan, "Modeling the diffusion of innovations using small-word networks," University of Kiel, Kiel, Germany, Working Paper, 2005.

[6] M. L. Bertotti and G. Modanese, "The bass diffusion model on finite barabasi-albert networks," Complexity, vol. 2019, Article ID 6352657, 12 pages, 2019.

[7] H. Batista da Silva, W. Uturbey, and B. M. Lopes, "Market diffusion of household PV systems: insights using the Bass model and solar water heaters market data," Energy for Sustainable Development, vol. 55, pp. 210-220, 2020.

[8] N. K. Dev, R. Shankar, and S. Swami, "Diffusion of green products in industry 4.0: reverse logistics issues during design of inventory and production planning system," International Journal of Production Economics, vol. 223, Article ID 107519, 2020.

[9] R. Kumar, A. K. Sharma, and K. Agnihotri, "Bifurcation analysis of a nonlinear diffusion model: effect of evaluation period for the diffusion of a technology," Arab Journal of Mathematical Sciences, vol. 25, no. 2, pp. 189-213, 2019.

[10] J. Massiani and A. Gohs, "The choice of Bass model coefficients to forecast diffusion for innovative products: an empirical investigation for new automotive technologies," Research in Transportation Economics, vol. 50, pp. 17-28, 2015.

[11] J. Grasman and M. Kornelis, "Forecasting product sales with a stochastic Bass model," Journal of Mathematics in Industry, vol. 9, no. 1, p. 2, 2019.

[12] S. Mitra, "Forecasting the diffusion of innovative products using the Bass model at the takeoff stage: a review of literature from subsistence markets," Asian Journal of Innovation and Policy, vol. 8, no. 1, pp. 141-161, 2019.

[13] S. Kim, K. Lee, J. K. Cho, and C. O. Kim, "Agent-based diffusion model for an automobile market with fuzzy TOPSISbased product adoption process," Expert Systems with Applications, vol. 38, no. 6, pp. 7270-7276, 2011.

[14] G. Aquino, M. Bologna, B. J. West, and P Grigolini, "Transmission of information between complex systems: $1 / f$ resonance," Physical Review. E, Statistical, Nonlinear, and Soft Matter Physics, vol. 83, Article ID 051130, 2011.

[15] Y. Peers, D. Fok, and P. H. Franses, "Modeling seasonality in new product diffusion," Marketing Science, vol. 31, no. 2, pp. 195-368, 2012.

[16] A. Galeotti, C. Ghiglino, and F. Squintani, "Strategic information transmission networks," Journal of Economic Theory, vol. 148, no. 5, pp. 1715-1769, 2013.

[17] R. Iyengar, C. Van den Bulte, and T. W. Valente, "Opinion leadership and social contagion in new product diffusion," Marketing Science, vol. 30, no. 2, pp. 195-212, 2011.

[18] T. Tashiro, "Hierarchical Bass model: a product diffusion model considering a diversity of sensitivity to fashion," 
Physica A: Statistical Mechanics and Its Applications, vol. 461, pp. 824-832, 2016.

[19] D. López-Pintado, "Diffusion in complex social networks," Games and Economic Behavior, vol. 62, no. 2, pp. 573-590, 2008.

[20] D. López-Pintado, "Influence networks," Games and Economic Behavior, vol. 75, no. 2, pp. 776-787, 2012.

[21] S. Li and Z. Jin, "Modeling and analysis of new products diffusion on heterogeneous networks," Journal of Applied Mathematics, vol. 2014, Article ID 940623, 12 pages, 2014.

[22] D. Horsky and L. S. Simon, "Advertising and the diffusion of new products," Marketing Science, vol. 2, no. 1, pp. 1-17, 1983.

[23] S. Swami and A. Dutta, "Advertising strategies for new product diffusion in emerging markets: propositions and analysis," European Journal of Operational Research, vol. 204, no. 3, pp. 648-661, 2010.

[24] Q. W. Huang and Y. L. Zhang, "Two competitive products diffusion in heterogeneous consumer social networks with repeat purchase," Advances in Difference Equations, vol. 309, 2019.

[25] R. Tuli, J. Dhar, and H. S. Bhatti, "Innovation diffusion model with interactions and delays in adoption for two competitive products in two different patches," Ricerche di Matematica, vol. 68, no. 2, pp. 705-726, 2019.

[26] G. Fu, F. Chen, J. Liu, and J. Han, "Analysis of competitive information diffusion in a group-based population over social networks," Physica A: Statistical Mechanics and Its Applications, vol. 525, pp. 409-419, 2019.

[27] A. M. A. Kermani, S. F. F. Ardestani, and F. Barzinpour, "A novel game theoretic approach for modeling competitive information diffusion in social networks with heterogeneous nodes," Physica A: Statistical Mechanics and Its Applications, vol. 466, pp. 570-582, 2017.

[28] S. Christian, K. Elmar, G. Markus et al., "Innovation diffusion of repeat purchase products in a competitive market: an agent-based simulation approach," European Journal of Operational Research, vol. 245, no. 1, pp. 157-167, 2015.

[29] J. Zhao, L. X. Yang, and X. Zhong, "Minimizing the impact of a rumor via isolation and conversion," Physica A: Statistical Mechanics and Its Applications, vol. 526, Article ID 120867, 2019.

[30] E. M. Stein and R. Shakarchi, Real Analysis: Measure Theory, Integration, and Hilbert Spaces, Princeton University Press, Princeton, NJ, USA, 2005.

[31] D. Liberzon, Calculus of Variations and Optimal Control Theory: A Concise Introduction, Princeton University Press, Princeton, NJ, USA, 2012.

[32] K. Atkinson, W. Han, and D. Stewart, Numerical Solution of Ordinary Differential Equation, Wiley, Hoboken, NJ, USA, 2009. 